\title{
Severe rhabdomyolysis without acute kidney injury in a female with acute dengue fever; an uncommon complication of a common disease
}

\author{
Senarathna HM${ }^{1}$, Abayagunawardana $\mathrm{AN}^{1}$, Weerarathna $\mathrm{TP}^{2}$ \\ ${ }^{I}$ University Medical Unit, Teaching Hospital Karapitiya, Galle, Sri Lanka. \\ ${ }^{2}$ Department of Medicine, Faculty of Medicine, University of Ruhuna, Galle, Sri Lanka. \\ Correspondence: Dr. H. M. Senarathna \\ e-mail: hmuthumal@gmail.com \\ (1) https://orcid.org/0000-0002-0523-5920
}

\section{Introduction}

Dengue fever (DF) is an emerging public health challenge particularly in developing countries including Sri Lanka. According to World Health Organiszation (WHO), about 100 million cases estimated to occur annually, putting almost half of the world's population at risk in over 100 tropical and sub-tropical countries (1).

Dengue is an arbo-virus of family Flaviviridae and there are 4 serotypes of the virus that cause dengue (DEN-1, DEN-2, DEN-3 and DEN-4). Its main vector is Aedes mosquito species, commonly A.aegypti and A.albopictus. Dengue viral infection can cause unpredicted, diverse clinical syndromes from simple flu like illness through dengue haemorrhagic fever (DHF) and dengue shock syndrome (DSS) to unusual organic manifestations like encephalopathy, myocarditis, hepatitis, acute kidney injury etc. (2).

Myalgia is a common symptom in dengue viral infection, but myositis and rhabdomyolysis are uncommon in DF and only a few case reports have been published in literature. Most of dengue related rhabdomyolysis have been complicated with acute kidney injury (3). We describe the case of a 26-year-old Sri Lankan female who developed rhabdomyolysis without acute kidney injury following dengue virus infection.

\section{Case presentation}

A 26-year-old previously healthy female, from dengue endemic area (Galle, Sri Lanka), presented with acute febrile illness with arthralgia, myalgia, and frontal headache, on the third day of illness. In addition, she also complained of severe back pain. She denied any exposure to suspect leptospirosis or typhus infections. Systemic inquiry was unremarkable. She has taken paracetamol $1 \mathrm{~g}$ only when she developed fever and not taken any other regular medication or illicit drugs previously. There was no family history of inherited muscle disorders. She is a teetotaler.

On admission she was ill looking, febrile $\left(102^{\circ} \mathrm{F}\right)$ and flushed. Her haemodynamic parameters were normal. There was mild tenderness over proximal muscle groups of upper and lower limbs. Muscle power was normal. Her lower back was extremely tender on palpation. Her respiratory, cardiovascular and abdominal examinations were unremarkable.

Initial full blood count revealed leucopenia and thrombocytopenia. Non-structural protein 1 antigen of dengue (NS1) was positive. On the next day, markedly elevated aminotransferases and creatine kinase (CK) were found, but liver functions and renal functions were within normal range (Table 1). Her urine was brown in color and positive for myoglobin. There were no red cells in urine on microscopic examination. Peripheral blood picture showed evidence of viral infection without haemolysis.

She was assessed every three hourly for haemodynamic parameters and urine output. She was given $125 \mathrm{ml}$ of fluid per hour (oral and Intravenous) with vigilance of fluid overload which was not apparent. During hospital stay her haemodynamic parameters did not deteriorate, haemoglobin and PCV were stable. Serial bed side focus ultrasonography did not reveal any features of fluid leakage. 
Table 1: Summary of investigations

\begin{tabular}{llccccccc}
\hline & & Day 3 & Day 4 & Day 5 & Day 6 & Day 7 & Day 8 & Day 15 \\
\hline WBC & $(\mathrm{x} \mathrm{10} / \mu \mathrm{L})$ & 2.6 & 3.92 & 4.48 & 6.99 & 7.48 & 7.7 & 7.66 \\
$\mathrm{SK}$ & $(\mu \mathrm{mol} / \mathrm{L})$ & - & 3.9 & 4.1 & 4.2 & 3.8 & 4.3 & 4.1 \\
$\mathrm{PT} / \mathrm{INR}$ & & - & 1.0 & - & 1.02 & - & 1.0 & - \\
$\mathrm{BIL}$ & $(\mu \mathrm{mol} / \mathrm{L})$ & - & 2.6 & - & 6 & - & 4.3 & - \\
$\mathrm{HBG}$ & $(\mathrm{g} / \mathrm{dl})$ & 14.5 & 13.3 & 12.9 & 12.8 & 11.9 & 12.6 & 12.6 \\
$\mathrm{SCR}$ & $(\mu \mathrm{mol} / \mathrm{L})$ & - & 44 & 45 & 43 & 41 & 43 & 42 \\
$\mathrm{ALB}$ & $(\mathrm{g} / \mathrm{L})$ & - & 36 & - & 34 & - & 33 & - \\
$\mathrm{PLT}$ & $(\mathrm{x} \mathrm{10} / \mu \mathrm{L})$ & 115 & 92 & 103 & 125 & 147 & 154 & 253 \\
$\mathrm{ALT}$ & $(\mathrm{U} / \mathrm{L})$ & 995 & 1023 & 1235 & 1392 & 1532 & 1482 & 269 \\
$\mathrm{AST}$ & $(\mathrm{U} / \mathrm{L})$ & 3278 & 3863 & 3924 & 4970 & 5186 & 3499 & 101 \\
$\mathrm{LDH}$ & $(\mathrm{U} / \mathrm{L})$ & - & 3522 & 4873 & & 2477 & 1193 & - \\
$\mathrm{CK}$ & $(\mathrm{U} / \mathrm{L})$ & - & 141960 & 242293 & 166339 & 92356 & 28183 & 1619 \\
\hline
\end{tabular}

HBG; Haemoglobin, WBC; White blood cells, PLT; Platelets, AST; Aspartate aminotransferase, ALT; Alanine aminotransferase, PT/INR; Prothrombin time-international normalize ratio, ALB; Albumin, BIL; Bilirubin, LDH; Lactate dehydrogenase,

CK; Creatine Kinase, SCR; Serum creatinine, SK; Serum Potassium

Her fever subsided on the fifth day after admission. The backache also improved permitting her to mobilize out of the bed. On the day six of illness IgM for dengue fever was also positive confirming the acute dengue viral infection. Throughout this period her urine output was satisfactory and renal functions were normal. Hepatitis B surface antigen (HBsAg), hepatitis $\mathrm{C}$ antibody and retroviral screening were negative. Serological test for leptospira was also negative.

She made a complete, uneventful recovery and was discharged from the hospital on day 8 of illness after assuring that transaminases and creatine kinase level coming down. She was advised to take more oral fluids and refrain from strenuous physical activity. One week after being discharged, her laboratory investigation showed further reduction of transaminases and CK.

\section{Discussion}

According WHO classification (4), diagnosis of dengue viral infection was firmly made in our patient who is from dengue endemic area and presented with acute febrile illness with arthralgia, myalgia, headache, leucopenia, thrombocytopenia and in whom both the NS1 antigen and dengue specific IgM antibody become positive.

Dengue can associate with many neuromuscular complications. Muscle involvement in patients with dengue infection can manifest with myalgias, myositis, rhabdomyolysis, and hypokalaemic paralysis (5). Myalgia affecting back and proximal limb muscles is one of the characteristic symptoms in dengue fever, particularly in febrile phase, and usually self-limiting. Dengue-associated myositis can range from self-limiting mild muscle weakness to severe dengue myositis which may cause death (5).

Rhabdomyolysis, often characterised by diffuse myalgia, elevated muscle enzymes and myoglobinuria, is the most devastating muscular manifestation of dengue viral infection. It is more common in male and in patients with $\operatorname{DHF}(5,6)$. Irrespective of CK level, it may be complicated with life threatening heme pigment-induced acute kidney injury (AKI) and associated electrolyte imbalances (7). AKI has developed with CK level 4,063 IU/L (8), as well as, 742,900 IU/L (9). Recent literature reviews have showed that most of the reported cases of dengue viral infection 
with rhabdomyolysis has been complicated with $\operatorname{AKI}(3,5)$.

Though the mechanism of muscle destruction in dengue viral infection is not very clear, it could be due to direct muscle invasion and replication of the virus and associated immune response (10). Huang, et al has also showed that interleukin-6 and tumour necrosis factor- $\alpha(\mathrm{TNF}-\alpha)$ levels were significantly increased in DHF with rhabdomyolysis than DHF without rhabdomyolysis in a small study (6).

A recent research in Taiwan has analysed 1,076 patients with dengue and found out that only 9 $(0.84 \%)$ had rhabdomyolysis. Of those patients who have developed rhabdomyolysis, 6 had acute kidney injury (6). Analysis of those 9 patients with rhabdomyolysis in comparison to the patient, who has not developed rhabdomyolysis, showed that hypertension, myalgia, and acute kidney injury were independent risk factors for developing rhabdomyolysis.

\section{Conclusions}

We herewith report a previously healthy female with serologically proven dengue fever complicated with severe rhabdomyolysis who made uneventful recovery without acute kidney injury. We highlight the need for clinical suspicion of this rare complication in patients with dengue fever who develop severe myalgia especially back pain and involving proximal muscles. Timely suspicion and screening with serum creatinine kinase level would help clinicians to take measures to prevent subsequent acute kidney injury.

Written informed consent was obtained from the patient for publication of this case report.

\section{References}

1. What is denguefl [Internet]. World Health Organization. World Health Organization; 2017 [cited 2020 May 09]. Available from: https://www.who.int/denguecontrol/ disease/en/.
2. World Health Organization, Special Programme for Research, Training in Tropical Diseases, World Health Organization. Department of Control of Neglected Tropical Diseases, World Health Organization. Epidemic, Pandemic Alert. Dengue; guidelines for diagnosis, treatment, prevention and control. World Health Organization; 2009.

3. Sargeant T, Harris T, Wilks R, Barned S, GallowayBlake K, Ferguson T. Rhabdomyolysis and dengue fever: a case report and literature review. Case reports in Medicine, 2013.

4. World Health Organization, Special Programme for Research, Training in Tropical Diseases, World Health Organization. Department of Control of Neglected Tropical Diseases, World Health Organization. Epidemic, Pandemic Alert. Dengue: guidelines for diagnosis, treatment, prevention and control. World Health Organization; 2009.

5. Garg RK, Malhotra HS, Jain A, Malhotra KP. Dengueassociated neuromuscular complications. Neurology India, 2015 Jul 1; 63(4): 497.

6. Huang SY, Lee K, Liu JW, Kung CT, Wang L. Clinical features of and risk factors for rhabdomyolysis among adult patients with dengue virus infection. The American Journal of Tropical Medicine and Hygiene, 2015 Jan 7; 92(1): 75-81.

7. Acharya S, Shukla S, Mahajan SN, Diwan SK. Acute dengue myositis with rhabdomyolysis and acute renal failure. Annals of Indian Academy of Neurology, $2010 \mathrm{Jul}$; 13(3): 221.

8. Repizo LP, Malheiros DM, Yu L, Barros RT, Burdmann EA. Biopsy proven acute tubular necrosis due to rhabdomyolysis in a dengue fever patient: a case report and review of literature. Revista do Instituto de Medicina Tropical de São Paulo, 2014 Feb; 56(1): 85-8.

9. Mok Y, Quah J, Siau C. A rare but potentially lethal complication of dengue. Asian Pacific Journal of Tropical Medicine, 2013 Jun 1; 6(6): 500-1.

10. Warke RV, Becerra A, Zawadzka A, Schmidt DJ, Martin KJ, Giaya K, Dinsmore JH, Woda M, Hendricks G, Levine T, Rothman AL. Efficient dengue virus (DENV) infection of human muscle satellite cells upregulates type I interferon response genes and differentially modulates MHC I expression on bystander and DENV-infected cells. Journal of General Virology, 2008 Jul 1; 89(7): 1605-15. 